Journal of Advanced Computer Science and Technology, 1 (4) (2012) 222-231

(C) Science Publishing Corporation

www.sciencepubco.com/index.php/JACST

\title{
Gradient Based Control and Synchronization of Lorenz System with Periodic Parametric Forcing
}

\author{
Mohammad Ali Khan \\ Department of Mathematics, Garhbeta Ramsundar Vidyabhaban \\ Paschim Medinipur, West Bengal, India. \\ Email: mdmaths@gmail.com
}

\begin{abstract}
Several important properties of chaos synchronization with gradient based control of chaotic system with periodic parametric forcing remain still unexplored. This paper investigates the behavior of the Lorenz system to change from chaotic to periodic, parametric forcing also entrains the system output with the forcing frequency. Secondly synchronization of two identically chaotic Lorenz systems are derived by linear feedback control and then discussed the gradient based control method for Lorenz system with periodic parametric forcing. Finally numerical simulation results are presented to show the feasibility and effectiveness of the approach.
\end{abstract}

Keywords: Chaotic systems, Gradient based control, Chaos synchronization, Periodic parametric forcing, Lorenz system.

\section{Introduction}

Experimental observations have pointed out that chaotic systems are common in nature. It is found that in Chemistry (Belouov-Zhabotinski reaction), in Nonlinear Optics (lasers), in Electronics (Chua-Matsumoto circuits), in Fluid Dynamics (Rayleigh-Benard convection), etc chaotic systems exist. Chaos is found in meteorology, solar system, heart and brain of living organisms and so on. Synchronization and control of interacting chaotic oscillators is one of the fundamental phenomena of nonlinear dynamics and chaos. Experimental realization of chaos synchronization and control have been achived 
with a magnetoelastic ribbon, a heart, a thermal convection loop, a diode oscillator, an optimal multimode chaotic solid-state laser, a Belousov-Zhabotinski reaction diffusion chemical system, and many other experiments.

One of the most striking discoveries in the study of chaos is that chaotic systems can be made to synchronize with each other. Synchronization of chaos is a phenomenon that may occur when two or more chaotic dynamical systems are coupled. This was discovered by Pecorra and carroll in 1990 [1]. Since Pecora and Carroll's [1] work many effective methods namely, OGY method [2], adaptive control [3], differential geometric method [4], inverse optimal control [5], lag synchronization [6], projective synchronization [7], anti-synchronization [8] etc for chaos control and synchronization have been proposed. Usually two dynamical systems are called synchronized if the distance between their corresponding states converges to zero as time goes to infinity. This type of synchronization is known as identical synchronization [1]. A generalization of the concept for unidirectionally coupled dynamical systems was proposed by Rulkov et al.[9], where two systems are called synchronized if a static functional relationship exists between the states of the systems. They called this kind of synchronization a generalized synchronization (GS). Kocarev and Parlitz [10] formulated a condition for the occurrence of GS between two coupled continuous dynamical systems. Yang and Chua [11] proposed GS of continuous dynamical systems via linear transformations. Tarai et.al.[12] introduce synchronization between two generalized bidirectionally coupled chaotic system. In 2011, Khan et.al. [13] have discussed three control strategies for unified chaotic system using dislocated feedback control, enhancing feedback control and speed feedback control. Recently Khan and Poria [14] have studied generalized synchronization of bidirectionally coupled chaotic systems. In 2006, Battogtokh et.al. [15] have discussed synchronization of eukaryotic cells by periodic forcing. Eccles et.al.[16] have studied synchronization and chaos control in a periodically forced quasi-geostropic two layer model of baroclinic instability in 2006. But no one discussed about gradient based control and synchronization of chaotic system with periodic parametric forcing.

Here we discuss in chapter 1, formalizes some of the important parameterdependent approaches, which typically include periodic parametric forcing. A general procedure, initiated by Ott.Grebogy and Yorke [2] is first to select a desired dynamical behavior from a variety of dynamics, including chaos and then to achieve and retain the behavior in some sense by applying tiny perturbations to the parameter of interest. The relative parameter variation on a system parameter, considered as a time-dependent, weak periodic oscillation in the form

$$
p \rightarrow p\left(1+\xi \psi_{p}(t)\right)
$$

where $\xi$ is a constant and $\psi_{p}(t)=\psi_{p}\left(t+t_{p}\right)$ is a parametric periodic function of period $t_{p}>0$. This type of coupling of weak periodic oscillations to a control 
parameter can be quite effective when applied to Lorenz chaotic system. In chapter 2, chaos synchronization uses two identically chaotic Lorenz systems via linear feedback control. Lastly, in chapter 3, we discusses one type of common adaptive control strategies, the gradient based adaptive control method for Lorenz system with parametric forcing. Finally numerical simulation results are presented to show the efficiency of our method.

\section{Lorenz system with parametrically forced convective flows}

The autonomous Lorenz system is the simplest model for the dynamics of convective layers and the dynamics of closed convection loops. Lorenz discovered that this simple-looking deterministic system could have extremely erratic dynamics, over a wide range of parameters, the solutions oscillate irregularly, never exactly repeating but always remaining in a bounded region of phase space. Now the Lorenz system is charaterized by the following differential equation

$$
\begin{aligned}
\dot{x} & =\sigma(y-x) \\
\dot{y} & =-x z-y+\left(r_{0}+r_{1} \cos (\omega t)\right) x \\
\dot{z} & =x y-b z
\end{aligned}
$$

where $\mathrm{x}, \mathrm{y}$ and $\mathrm{z}$ are state variables and $\sigma, r_{0}, r_{1}, \omega, b>0$ are parameters. The parametric forcing term $r_{0}+r_{1} \cos (\omega t)$ is a periodic Rayleigh number, proportional to the temperature drop between the bottom and the top boundaries. This parametrically forced model can be understand as the convective fluid being heated periodically at its bottom or cooled periodically at its top to control the motion of the fluid and the heat transfer within it. It can be shown from Figure 1-Figure 4, that periodic parametric forcing causes the Lorenz system to undergo a transition from being chaotic to being one-periodic, two-periodic and then four-periodic. Further increasing the mean value of the Rayleigh number $r_{0}$ will continue this periodic-doubling process until a new chaotic attractor appears. This experiment clearly shows how simple parametric forcing makes it possible to achieve a controlled transition to chaos, by simply varying the mean value of the forcing term.

\section{Synchronization of identical Lorenz system with periodic forcing via linear feedback}

Synchronization of the drive-response system can be understand from a feedback control point of view. To simulate this type of synchronization via linear 


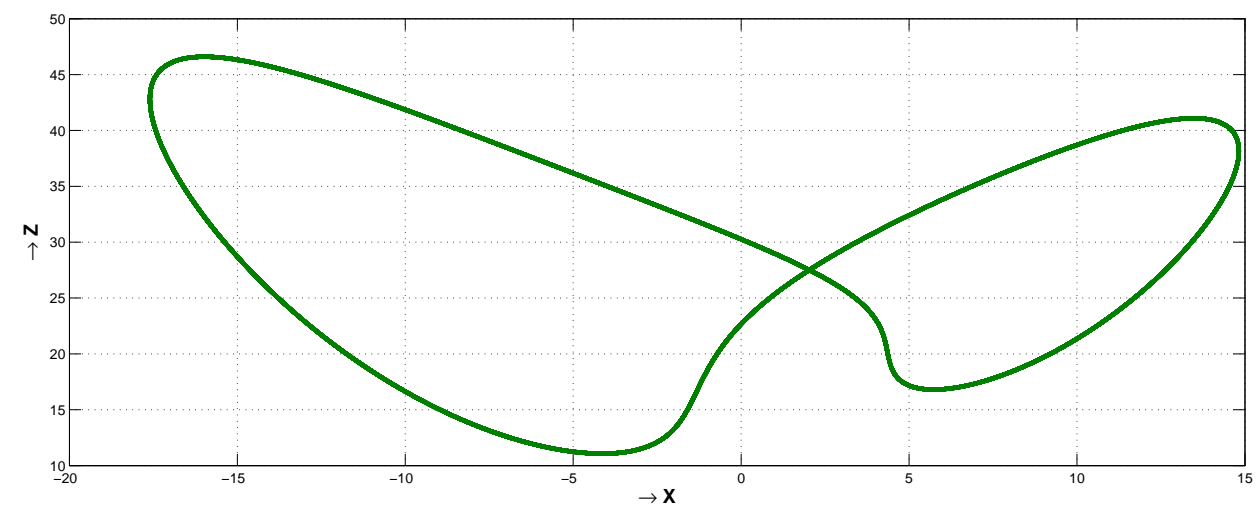

Figure 1: Period-one with periodic parametric forcing $r_{0}=26.5$ and $r_{1}=5$

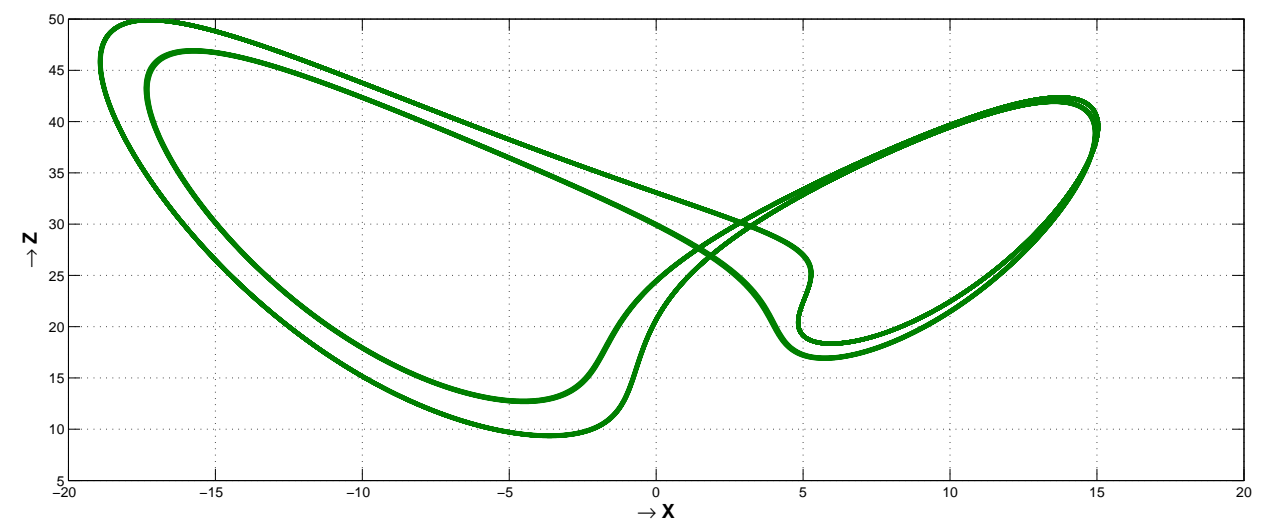

Figure 2: Period-two with periodic parametric forcing $r_{0}=27.5$ and $r_{1}=5$ 


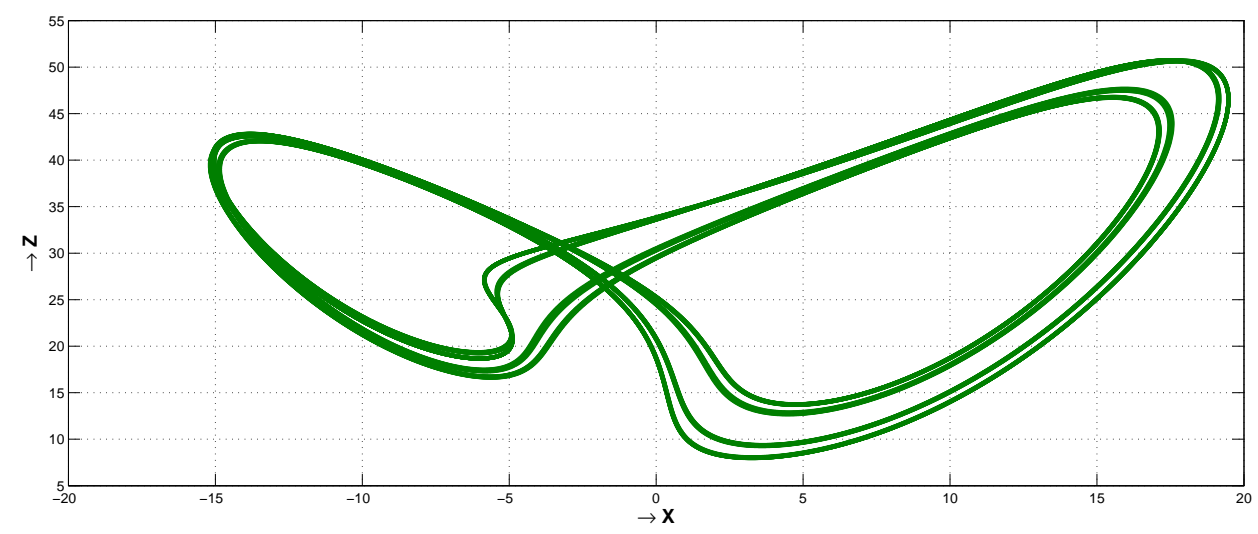

Figure 3: Period-four with periodic parametric forcing $r_{0}=27.9$ and $r_{1}=5$

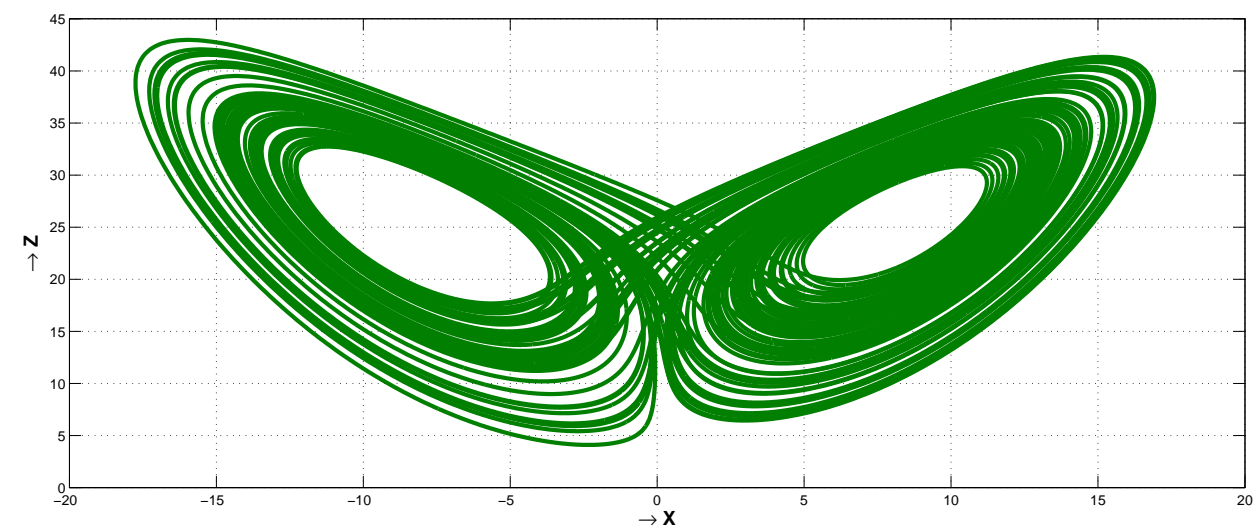

Figure 4: Chaos with periodic parametric forcing $r_{0}=26.5$ and $r_{1}=0$ 


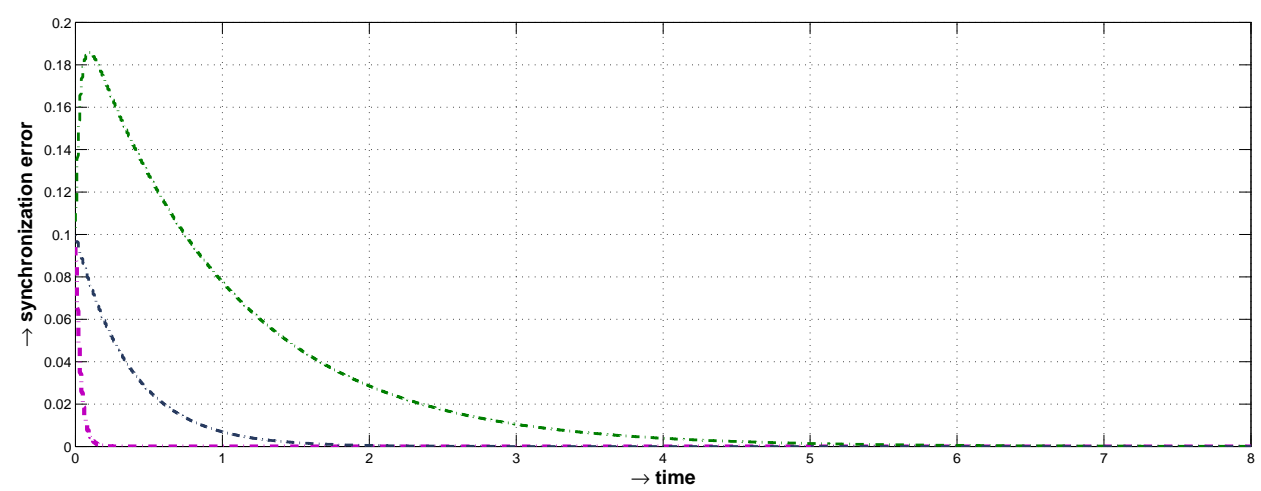

Figure 5: Synchronization error with linear feedback controller coefficient $K_{x}=$ 20.0, $K_{y}=\sigma=10.0$ and $K_{z}=40.0$

feedback, may chaotic systems can be used, including the familier Lorenz system. When Lorenz system with parametric forcing is used then drive and response system becomes

$$
\begin{aligned}
\dot{x_{1}} & =\sigma\left(y_{1}-x_{1}\right) \\
\dot{y_{1}} & =\left(r_{0}+r_{1} \cos (\omega t)\right) x_{1}-y_{1}-x_{1} z_{1} \\
\dot{z_{1}} & =x_{1} y_{1}-b z_{1}
\end{aligned}
$$

and

$$
\begin{aligned}
\dot{x_{2}} & =\sigma\left(y_{2}-x_{2}\right)+K_{x}\left(x_{1}-x_{2}\right)-K_{y}\left(y_{1}-y_{2}\right) \\
\dot{y_{2}} & =\left(r_{0}+r_{1} \cos (\omega t)\right) x_{2}-y_{2}-x_{2} z_{2}+K_{z}\left(z_{1}-z_{2}\right) \\
\dot{z_{2}} & =x_{2} y_{2}-b z_{2}
\end{aligned}
$$

Substracting equation (2) from equation (3) yields the error dynamical system as

$$
\begin{aligned}
& \dot{e_{1}}=-\left(\sigma+K_{x}\right) e_{1}+\left(\sigma-K_{y}\right) e_{2} \\
& \dot{e_{2}}=\left(r_{0}+r_{1} \cos (\omega t)\right) e_{1}-e_{2}-K_{z} e_{3}+x_{1} z_{1}-x_{2} z_{2} \\
& \dot{e_{3}}=-b e_{3}+x_{2} y_{2}-x_{1} y_{1}
\end{aligned}
$$

where $e_{1}=x_{2}-x_{1}, e_{2}=y_{2}-y_{1}$ and $e_{3}=z_{2}-z_{1}$. Therfore the states of the response system (3) and the states of the drive system (2) are globally synchronized asympotically i.e.

$$
\lim _{t \rightarrow \infty}\|e(t)\|=0
$$

for $\sigma=10.0, b=8 / 3, r_{0}=27.5, r_{1}=5.0$ and with feedback controller coefficient $K_{x}=20.0, K_{y}=\sigma=10.0$ and $K_{z}=40.0$ where $e(t)=\left[e_{1}, e_{2}, e_{3}\right]^{T}$. 


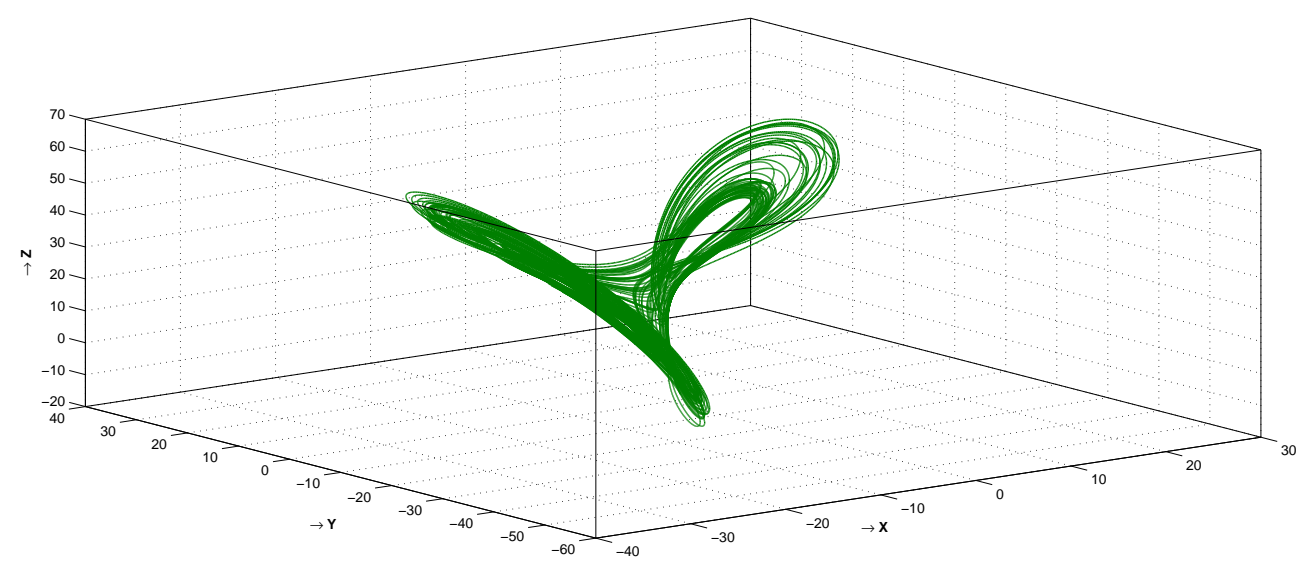

Figure 6: Gradient based control of chaotic Lorenz system

\section{Gradient based control of Lorenz system with parametric forcing}

To illustrate the gradient based adaptive control method, consider the chaotic Lorenz system (1). The objective is to control its trajectory to an unstable equilibrium $\left(\xi, \xi, \frac{\xi^{2}}{b}\right)$ where $\xi^{2}=b(r-1)$. A control input $u=\left[u_{1}, u_{2}, u_{3}\right]^{T}$ is added to the system (1). Then the Lorenz system becomes

$$
\begin{aligned}
\dot{x} & =\sigma(y-x)+u_{1} \\
\dot{y} & =-x z-y+\left(r_{0}+r_{1} \cos (\omega t)\right) x+u_{2} \\
\dot{z} & =x y-b z+u_{3}
\end{aligned}
$$

Let $e_{x}=x-\xi, e_{y}=y-\xi$ and $e_{z}=z-\frac{\xi^{2}}{b}$, therefore the error dynamical system becomes

$$
\begin{aligned}
\dot{e_{x}} & =\sigma\left(e_{y}-e_{x}\right)+u_{1} \\
\dot{e_{y}} & =\left[\left(r_{0}+r_{1} \cos (\omega t)\right)-\frac{\xi^{2}}{b}\right] e_{x}-e_{y}-\xi e_{z}-e_{x} e_{z}+\xi\left(r_{0}+r_{1} \cos (\omega t)\right)-\frac{\xi^{3}}{b}-\xi+u_{2} \\
\dot{e_{z}} & =\xi\left(e_{x}+e_{y}\right)-b e_{z}+e_{x} e_{y}+u_{3}
\end{aligned}
$$

Let us consider the resulting controller as

$$
u=\left[\begin{array}{ll}
0 & (x-\xi) u_{0}+\xi\left(u_{0}+2\right) \quad \xi(a-\xi)
\end{array}\right]^{T} \text { where } u_{0}=\left(\frac{\xi^{2}}{b}-r-1\right) .
$$

Then the system becomes

$$
\dot{e_{x}}=\sigma\left(e_{y}-e_{x}\right)
$$




$$
\begin{aligned}
& \dot{e_{y}}=-e_{x}-e_{y}-\xi e_{z}-e_{x} e_{z} \\
& \dot{e_{z}}=\xi e_{y}-b e_{z}+e_{x} e_{y}
\end{aligned}
$$

The objective function is chosen as

$$
F_{0}(x, t)=\frac{1}{2}\left(\frac{1}{\sigma} e_{x}^{2}+e_{y}^{2}+e_{z}^{2}\right)
$$

then $\dot{F}_{0}=-e_{x}^{2}-e_{y}^{2}-b e_{z}^{2}<0$. Here $u_{0}=\left(\frac{\xi^{2}}{b}-r-1\right)$ is the ideal controller of the gradient based controller of Lorenz system.

\section{$5 \quad$ Numerical Results}

Numerical simulations are done by Fourth order Runge-Kutta method. We consider $\sigma=10, \omega=7.62$ and $b=8 / 3$ are fixed, $r_{0}$ is varied from 26.5 to 27.5 , then to 27.9 , with $r_{1}=0$ and then changed to $r_{1}=5.0$. From Fig.1Fig. 4 it is clear that periodic parametric forcing causes the Lorenz system to undergo a transition from being chaotic to being one-periodic, two periodic and then four chaotic. We assume that the initial values of the error as $e_{1}(0)=$ $0.1, e_{2}(0)=0.1$, and $e_{3}(0)=0.1$. Picture shows that the error is going to zero asymptotically i.e. to two chaotic Lorenz systems with periodic parametric forcing synchronized. Numerical simulation results is shown for gradient based control of Lornz chaotic system with $\sigma=10, r_{0}=27.5, r_{1}=5.0$ and $b=8 / 3$.

\section{Conclusion}

In this work, gradient based control and synchronization of Lorenz system with periodic parametric forcing is achieved. Numerical simulations are shown to be consistent with theoretical statements. Lorenz system is taken an example to show the effectiveness of our results. This synchronization scheme may be useful for secure communication and for biological purposes.

\section{ACKNOWLEDGEMENTS}

I am grateful to the Editor for careful reading and constructive comments.

\section{References}

[1] Pecorra and Carroll, Synchronization in chaotic systems, Phys.Rev.Lett., $64(1990) 821-824$.

[2] Ott, Grebogi, Yorke, Controlling chaos, Phys.Rev.Lett., 64 (1990)11961199. 
[3] Lu.Chen, Synchronization of an uncertain unified chaotic system via adaptive control, Chaos, Soliton and Fractals, 14 (2002)643.

[4] C.C.Fuh and P.C.Tung, Controlling chaos using differential geometric method, Phys.Rev.Lett., 75 (1995)2952.

[5] E.N.Sanchez, J.P.Perez, M.Martinez and G.Chen, Chaos stabilization: an inverse optimal control approach, Latin Am Appl Res:Int J 32 (2002)111.

[6] A. Tarai, S.Poria and P. Chatterjee, Synchronization of bidirectionally coupled chaotic Chen's system with delay, Chaos Solitons and Fractals., 41 (2009)643-647.

[7] R. Mainieri and J. Rahacek, Projective synchronization in three dimensional chaotic systems, Phys. Rev. Lett., 82 (1999)3042-3045.

[8] M.A. Khan, S.N. Pal and S. Poria, Generalized anti-synchronization of different chaotic systems, Int. J. of Applied Mechanics and Engineering, 17(2012)83-89.

[9] N.F.Rulkov, M.M.Sushchik, L.S.Tsimring and H.D.I Abarbanel, Generalized synchronization of chaos in directionally coupled chaotic systems, Phys. Rev.E., 51 (1995)980-994.

[10] L.Kocarev and U.Parlitz, Generalized synchronization, predictability, an equivalence of unidirectionally coupled dynamical systems, Phys.Rev.Lett. 76 (1996)1816-1819.

[11] T.Yang and L.O.Chua, Generalized synchronization of chaos via linear transformations, International Journal of Bifurcation and Chaos,9 (1999)215-219

[12] A. Tarai, S.Poria and P. Chatterjee, Synchronization of generalised linearly bidirectionally coupled unified system, Chaos Solitons and Fractals., 40 (2009)885-892.

[13] M.A. Khan, A.K. Mondal and S. Poria, Three control strategies for unified chaotic system, Int. J. of Applied Mechanics and Engineering, 16 (2011)597-608.

[14] Mohammad Ali Khan and Swarup Poria, Generalized synchronization of bidirectionally coupled chaotic systems, Int. J. of Applied Mathematical Research, 1 (2012)303-313.

[15] D. Battogtokh, K. Ajhara and JJ. Tyson, Synchronization of eukaryotic cells by periodic forcing, Phys. Rev. Lett., 96 (2006)148102. 
[16] F.J.R. Eccles, P.L. Read and T.W.N. Hains, Synchronization and chaos control in a periodically forced quasi-geostropic two layer model of baroclinic instability, Nonlin. Processes Geophysics., 13 (2006)23-39. 